\title{
OPTIMAL CONTROL OF A ROTATING BODY BEAM*
}

\author{
WEIJIU LIU ${ }^{1}$
}

\begin{abstract}
In this paper we consider the problem of optimal control of the model for a rotating body beam, which describes the dynamics of motion of a beam attached perpendicularly to the center of a rigid cylinder and rotating with the cylinder. The control is applied on the cylinder via a torque to suppress the vibrations of the beam. We prove that there exists at least one optimal control and derive a necessary condition for the control. Furthermore, on the basis of iteration method, we propose numerical approximation scheme to calculate the optimal control and give numeric examples.
\end{abstract}

Mathematics Subject Classification. 49K20, 35L75, 74K10.

Received August 2, 2000. Revised February 26 and July 20, 2001.

\section{INTRODUCTION}

The purpose of this paper is to study the optimal control of the model for a rotating body beam (see, e.g. $[2,3,6,15])$

$$
\begin{array}{ll}
u_{t t}(x, t)+u_{x x x x}(x, t)=\omega^{2}(t) u(x, t) & \text { in }(0,1) \times(0, T), \\
\frac{\mathrm{d}}{\mathrm{d} t}\left[\omega(t)\left(1+\int_{0}^{1} u^{2}(x, t) \mathrm{d} x\right)\right]=\gamma(t) & \text { in }(0, T), \\
u(0, t)=u_{x}(0, t)=u_{x x}(1, t)=u_{x x x}(1, t)=0 & \text { in }(0, T), \\
u(x, 0)=u^{0}(x), u_{t}(x, 0)=u^{1}(x), \omega(0)=\omega^{0} & \text { in }(0,1) .
\end{array}
$$

In (1.1-1.4), the subscripts denote the derivatives with respect to the time variable $t$ or the space variable $x$, $\omega(t)=\dot{\theta}(t)$ is the angular velocity of the cylinder at time $t, u(x, t)$ is the beam's displacement in the rotating plane at time $t$ and point $x$ and $\gamma(t)$ is the torque control variable applied to the cylinder at time $t$. This model describes the dynamics of motion of a beam attached perpendicularly to the center of a rigid cylinder and rotating with the cylinder (see Fig. 1.1). As explained in $[2,4]$, it has applications in aerospace engineering. Indeed, we can imagine Figure 1.1 a satellite, the beam being its antenna.

As the cylinder rotates, the beam deviates from its equilibrium and becomes unstable. So control mechanisms are needed to stabilize it. Indeed, extensive attention has been paid to the problem of stabilization for (1.1-1.4). Applying the Lyapunov analysis and backstepping method, feedback torque control laws were proposed (see,

Keywords and phrases: Rotating body beam, optimal control, numerical approximation scheme.

* Supported by the Killam Postdoctoral Fellowship.

${ }^{1}$ Department of Mathematics and Statistics, Dalhousie University, Halifax, Nova Scotia, B3H 3J5, Canada;

e-mail: weiliu@mscs.dal.ca

(c) EDP Sciences, SMAI 2002 


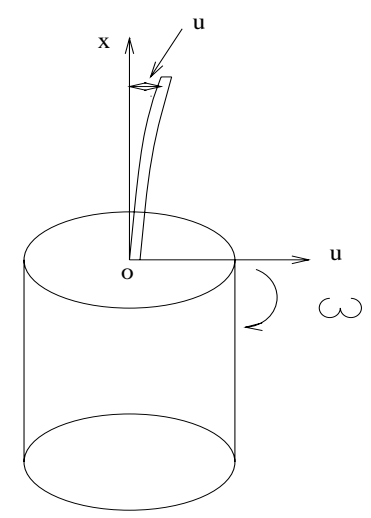

Figure 1.1. A rotating body beam.

e.g. $[5,6,9,15])$ to globally asymptotically stabilize the equilibrium point $(0, \bar{\omega})$ provided

$$
\bar{\omega} \in\left(-\omega_{c}, \omega_{c}\right)
$$

where $\omega_{c}$ is an explicit critical angular velocity (see, e.g. $[6,15]$ ). It was also proved in [15] that there is no stabilizing control law if $|\omega| \geq \omega_{c}$. This is physically reasonable because if the cylinder spins too fast the beam shall be out of control and can not be stabilized by feedback control laws. In the above problem of stabilization, it was required that the stabilizing control laws drive the beam eventually to its equilibrium, that is, $\lim _{t \rightarrow \infty} u(t, x)=0$. Such a requirement is quite strict. So we relax it and we just require controls to drive the beam to approach its equilibrium as closely as possible. Therefore, we consider the problem of optimal control with the cost function defined by

$$
J(\gamma)=\int_{0}^{T} \int_{0}^{1}\left[u^{2}(x, t, \gamma)+(\omega(t, \gamma)-\bar{\omega})^{2}\right] \mathrm{d} x \mathrm{~d} t+\int_{0}^{T} \gamma^{2}(t) \mathrm{d} t
$$

In this case, the angular velocity $\bar{\omega}$ can be an arbitrary number. We might want to consider a cost function over an infinite time interval

$$
J_{\infty}(\gamma)=\int_{0}^{\infty} \int_{0}^{1}\left[u^{2}(x, t, \gamma)+(\omega(t, \gamma)-\bar{\omega})^{2}\right] \mathrm{d} x \mathrm{~d} t+\int_{0}^{\infty} \gamma^{2}(t) \mathrm{d} t
$$

This cost function does make more sense than $J(\gamma)$ as the beam is needed to be controlled over a long time, not a short time, in reality. However $J_{\infty}(\gamma)$ is difficult to be handled as it is not clear whether $J_{\infty}(\gamma)$ is proper, that is, $J_{\infty}(\gamma)<\infty$ for some $\gamma$.

Concerning the optimal control for large space structures, important results have been established (see, e.g. $[4,7]$ ). Indeed, Biswas and Ahmed [4] addressed the model for a spacecraft consisting of a rigid bus and a flexible beam and developed necessary conditions for determining the control torque and forces for optimal regulation of attitude maneuvers of the spacecraft along with simultaneous suppression of elastic vibrations of the flexible beam.

Let $\Gamma$ be a closed and convex subset of $L^{2}(0, T)$ (for the notation appearing in the introduction, see the next section). The optimal control problem of $(1.1-1.4)$ is to minimize $J(\gamma)$

$$
\inf _{\gamma \in \Gamma} J(\gamma)
$$


This means that we want to find a torque control $\gamma$ of the least cost to drive the system to approach the equilibrium point $(0, \bar{\omega})$ as closely as possible. Any element $\gamma_{o}$ such that

$$
J\left(\gamma_{o}\right)=\inf _{\gamma \in \Gamma} J(\gamma)
$$

is called a solution of problem (1.8). The element $\gamma_{o}$ is termed an optimal control, the corresponding state $\left(u\left(\gamma_{o}\right), \omega\left(\gamma_{0}\right)\right)$ termed an optimal state and the pair $\left(\gamma_{o}, u\left(\gamma_{o}\right), \omega\left(\gamma_{0}\right)\right)$ termed an optimal pair. We call the subset $\Gamma$ a set of admissible controls.

We are also interested in an optimal obstacle problem. We can imagine that any controls are subject to certain circumstances such as physical locations and available resources, which obstruct us to implement the controls arbitrarily. For instance, when we use a unidirectional jet to implement the torque control, the control would be subject to certain constraint of a limited range, e.g. $\gamma \geq 0$. So we introduce the following obstacle problem. For any $\theta \in L^{2}(0, T)$, we denote

$$
\Gamma(\theta)=\left\{\gamma \in L^{2}(0, T): \gamma \geq \theta \text { a.e. on }(0, T)\right\}
$$

We call $\theta$ an obstacle. If $\theta \equiv 0$, this means that the torque control is implemented by a unidirectional jet. We can imagine that different jets would produce different obstacles $\theta$. The problem of optimal obstacle is to find a $\theta_{o}$ (corresponding to an optimal jet) such that

$$
\inf _{\gamma \in \Gamma\left(\theta_{o}\right)} J(\gamma) \leq \inf _{\gamma \in \Gamma(\theta)} J(\gamma) \quad \forall \theta \in L^{2}(0, T)
$$

If we define the cost function $F(\theta)$ by

$$
F(\theta)=\inf _{\gamma \in \Gamma(\theta)} J(\gamma)
$$

then optimal obstacle problem (1.10) becomes the following minimization problem

$$
\inf _{\theta \in L^{2}(0, T)} F(\theta)
$$

We note that the obstacle $\theta$ is not taken into account in (1.12), that is, while we try to minimize the pair $(u, \omega, \gamma)$, the obstacle $\theta$ may be large. To ensure that the obstacle is not too large, we introduce the following cost funtion $G(\theta)$

$$
G(\theta)=F(\theta)+\int_{0}^{T} \theta^{2}(t) \mathrm{d} t
$$

and minimize it:

$$
\inf _{\theta \in L^{2}(0, T)} G(\theta) .
$$

To further minimize the corresponding state $(u(\theta), \omega(\theta))$, we can consider the cost function

$$
H(\theta)=F(\theta)+J(\theta)
$$

and the minimization problem

$$
\inf _{\theta \in L^{2}(0, T)} H(\theta) .
$$


Applying the theory of semigroups, we prove that problem (1.1-1.4) is well posed in Section 2. We then solve optimal control problems $(1.8,1.12,1.14)$ and $(1.16)$ by employing the theory of optimization in Section 3. On the basis of the iteration method, we propose a numerical approximation scheme to calculate the optimal control in Section 4.

\section{Global Strong SOlutions}

We now introduce notation used throughout the paper. For $-\infty<a<b<\infty$, we denote by $H^{s}(a, b)$ the usual Sobolev space (see $[1,11]$ ) for any $s \in \mathbb{R}$. For $s \geq 0, H_{0}^{s}(a, b)$ denotes the completion of $C_{0}^{\infty}(a, b)$ in $H^{s}(a, b)$, where $C_{0}^{\infty}(a, b)$ denotes the space of all infinitely differentiable functions on $(a, b)$ with compact support in $(a, b)$. Set

$$
\begin{aligned}
H_{0^{-}}^{2}(0,1) & =\left\{\varphi \in H^{2}(0,1): \varphi(0)=\varphi_{x}(0)=0\right\} \\
H_{0^{-}}^{4}(0,1) & =\left\{\varphi \in H^{4}(0,1): \varphi(0)=\varphi_{x}(0)=\varphi_{x x}(1)=\varphi_{x x x}(1)=0\right\} \\
\mathcal{H} & =H_{0^{-}}^{2}(0,1) \times L^{2}(0,1) .
\end{aligned}
$$

We use the following $H^{2}$ norm of $H_{0^{-}}^{2}(0,1)$

$$
\|\varphi\|_{H^{2}}=\left(\int_{0}^{1} \varphi_{x x}^{2} \mathrm{~d} x\right)^{1 / 2}, \varphi \in H_{0^{-}}^{2}(0,1)
$$

which are equivalent to the usual one. The norm on $L^{2}(0,1)$ is denoted by $\|\cdot\|$. Let $X$ be a Banach space. We denote by $C^{n}([0, T] ; X)$ the space of $n$ times continuously differentiable functions defined on $[0, T]$ with values in $X$, and write $C([0, T] ; X)$ for $C^{0}([0, T] ; X)$.

Applying the theory of semigroups, we prove that problem (1.1-1.4) is well posed. For the definitions of mild and strong solutions below, we refer to [12] (p. 106), Definition 2.3 (p. 109), Definition 2.8. The following theorem is a slight extension of Lemma 1 of [15] and its proof is similar.

Theorem 2.1. (i) For the initial condition $\left(u^{0}, u^{1}, \omega^{0}\right) \in \mathcal{H} \times \mathbb{R}$, control $\gamma \in L^{1}(0, T)$ and any $T>0$, problem (1.1-1.4) has a unique mild solution satisfying

$$
\begin{aligned}
u=u(x, t, \gamma) & \in C\left([0, T] ; H_{0^{-}}^{2}((0,1)) \cap C^{1}\left([0, T] ; L^{2}(0,1)\right),\right. \\
\omega=\omega(x, t, \gamma) & \in C([0, T] ; \mathbb{R}) .
\end{aligned}
$$

Moreover, for two solutions $\left(u_{1}, \omega_{1}\right)$ and $\left(u_{2}, \omega_{2}\right)$ of (1.1-1.4) corresponding to $\left(u_{1}^{0}, u_{1}^{1}, \omega_{1}^{0}\right) \in \mathcal{H} \times \mathbb{R}, \gamma_{1} \in$ $L^{1}(0, T)$ and $\left(u_{2}^{0}, u_{2}^{1}, \omega_{2}^{0}\right) \in \mathcal{H} \times \mathbb{R}, \gamma_{2} \in L^{1}(0, T)$, respectively, there exists a positive constant $C=C\left(\left\|u_{1}^{0}\right\|,\left|\omega_{1}^{0}\right|\right.$, $\left.\left\|\gamma_{1}\right\|_{L^{1}(0, T)},\left\|u_{2}^{0}\right\|,\left|\omega_{2}^{0}\right|,\left\|\gamma_{2}\right\|_{L^{1}(0, T)}, T\right)$, continuously depending on its arguments, such that for $0 \leq t \leq T$

$$
\begin{aligned}
& \left\|u_{1 x x}(t)-u_{2 x x}(t)\right\|+\left\|u_{1 t}(t)-u_{2 t}(t)\right\|+\left|\omega_{1}(t)-\omega_{2}(t)\right| \\
& \leq C\left(\left\|u_{1 x x}^{0}-u_{2 x x}^{0}\right\|+\left\|u_{1}^{1}-u_{2}^{1}\right\|+\left|\omega_{1}^{0}-\omega_{2}^{0}\right|+\left\|\gamma_{1}-\gamma_{2}\right\|_{L^{1}(0, T)}\right) .
\end{aligned}
$$

(ii) For the initial condition $\left(u^{0}, u^{1}, \omega^{0}\right) \in H_{0^{-}}^{4}(0,1) \times H_{0^{-}}^{2}(0,1) \times \mathbb{R}$ and $\gamma \in C[0, T]$, problem (1.1-1.4) has a unique strong solution satisfying

$$
\begin{aligned}
u & \in L^{1}\left((0, T) ; H_{0^{-}}^{4}((0,1))\right) \cap C\left([0, T] ; H_{0^{-}}^{2}((0,1)),\right. \\
u_{t} & \in L^{1}\left((0, T) ; H_{0^{-}}^{2}((0,1))\right) \cap C\left([0, T] ; L^{2}(0,1)\right), \\
u_{t t} & \in L^{1}\left((0, T) ; L^{2}(0,1)\right) \\
\omega & \in C^{1}([0, T] ; \mathbb{R}) .
\end{aligned}
$$


Proof. (i) Integrating (1.2) from 0 to $t$, we obtain

$$
\omega(t)=\frac{\int_{0}^{t} \gamma(s) \mathrm{d} s+a_{0}}{1+\|u(t)\|^{2}}
$$

where

$$
a_{0}=\omega^{0}\left(1+\left\|u^{0}\right\|^{2}\right)
$$

Substituting $\omega$ into (1.1), we obtain

$$
\begin{array}{ll}
u_{t t}(x, t)+u_{x x x x}(x, t)=\frac{\left(\int_{0}^{t} \gamma(s) \mathrm{d} s+a_{0}\right)^{2} u(x, t)}{\left(1+\|u(t)\|^{2}\right)^{2}} & \text { in }(0,1) \times(0, T), \\
u(0, t)=u_{x}(0, t)=u_{x x}(1, t)=u_{x x x}(1, t)=0 & \text { in }(0, T), \\
u(x, 0)=u^{0}(x), u_{t}(x, 0)=u^{1}(x) & \text { in }(0,1) .
\end{array}
$$

Set

$$
f\left(t, \varphi, \gamma, a_{0}\right)=\frac{\left(\int_{0}^{t} \gamma(s) \mathrm{d} s+a_{0}\right)^{2} \varphi(x)}{\left(1+\|\varphi\|^{2}\right)^{2}}
$$

One can readily verify that, for any fixed $\gamma \in L^{1}(0, T)$ and $a_{0}, f:[0, T] \times L^{2}(0,1) \rightarrow L^{2}(0,1)$ is continuous in $t$ on $[0, T]$ and uniformly Lipschitz continuous on $L^{2}(0,1)$. Moreover, it is well known that the operator $A$ defined by

$$
A(\varphi, \psi)=\left(\psi,-\varphi_{x x x x}\right)
$$

with domain $D(A)=H_{0^{-}}^{4}(0,1) \times H_{0^{-}}^{2}(0,1)$ generates a $C_{0}$ semigroup $S(t)$ on $H_{0^{-}}^{2}(0,1) \times L^{2}(0,1)$. Therefore, by the classical theory of semigroups (see, e.g. [12], p. 184, Th. 1.2), problem (2.14-2.16) has a unique mild solution with

$$
u \in C\left([0, T] ; H_{0^{-}}^{2}((0,1)) \cap C^{1}\left([0, T] ; L^{2}(0,1)\right) .\right.
$$

Since

$$
\omega(t)=\frac{\int_{0}^{t} \gamma(s) \mathrm{d} s+a_{0}}{1+\|u(t)\|^{2}}
$$

problem (1.1-1.4) has a unique mild solution satisfing (2.5) and (2.6).

Let $\left(u_{1}, \omega_{1}\right)$ and $\left(u_{2}, \omega_{2}\right)$ be two solutions of (1.1-1.4) corresponding to $\left(u_{1}^{0}, u_{1}^{1}, \omega_{1}^{0}\right) \in \mathcal{H} \times \mathbb{R}, \gamma_{1} \in L^{1}(0, T)$ and $\left(u_{2}^{0}, u_{2}^{1}, \omega_{2}^{0}\right) \in \mathcal{H} \times \mathbb{R}, \gamma_{2} \in L^{1}(0, T)$, respectively. Set

$$
y=u_{1}-u_{2} .
$$

Then we have

$$
y_{t t}+y_{x x x x}=f\left(t, u_{1}(x, t), \gamma_{1}, a_{1}\right)-f\left(t, u_{2}(x, t), \gamma_{2}, a_{2}\right),
$$


where

$$
\begin{aligned}
& a_{1}=\omega_{1}^{0}\left(1+\left\|u_{1}^{0}\right\|^{2}\right), \\
& a_{2}=\omega_{2}^{0}\left(1+\left\|u_{2}^{0}\right\|^{2}\right) .
\end{aligned}
$$

Multiplying $(2.21)$ by $y_{t}$ and integrating over $(0,1)$ by parts, we obtain

$$
\frac{\mathrm{d}}{\mathrm{d} t}\left(\left\|y_{t}(t)\right\|^{2}+\left\|y_{x x}(t)\right\|^{2}\right)=2 \int_{0}^{1}\left[f\left(t, u_{1}(x, t), \gamma_{1}, a_{1}\right)-f\left(t, u_{2}(x, t), \gamma_{2}, a_{2}\right)\right] y_{t}(x, t) \mathrm{d} x
$$

In what follows, $C=C\left(\left\|u_{1}^{0}\right\|,\left|\omega_{1}^{0}\right|,\left\|\gamma_{1}\right\|_{L^{1}(0, T)},\left\|u_{2}^{0}\right\|,\left|\omega_{2}^{0}\right|,\left\|\gamma_{2}\right\|_{L^{1}(0, T)}, T\right)$ denotes a generic positive constant which continuously depends on its arguments and may vary from line to line. Since

$$
\begin{aligned}
& \left|f\left(t, u_{1}(x, t), \gamma_{1}, a_{1}\right)-f\left(t, u_{2}(x, t), \gamma_{2}, a_{2}\right)\right| \\
& =\left|\frac{\left(\int_{0}^{t} \gamma_{1}(s) \mathrm{d} s+a_{1}\right)^{2} u_{1}(x, t)}{\left(1+\left\|u_{1}(t)\right\|^{2}\right)^{2}}-\frac{\left(\int_{0}^{t} \gamma_{2}(s) \mathrm{d} s+a_{2}\right)^{2} u_{2}(x, t)}{\left(1+\left\|u_{2}(t)\right\|^{2}\right)^{2}}\right| \\
& \leq \frac{\left(\int_{0}^{t} \gamma_{1}(s) \mathrm{d} s+a_{1}\right)^{2}\left|u_{1}(x, t)-u_{2}(x, t)\right|}{\left(1+\left\|u_{1}(t)\right\|^{2}\right)^{2}} \\
& +\left|u_{2}(x, t)\right|\left|\frac{\left(\int_{0}^{t} \gamma_{1}(s) \mathrm{d} s+a_{1}\right)^{2}}{\left(1+\left\|u_{1}(t)\right\|^{2}\right)^{2}}-\frac{\left(\int_{0}^{t} \gamma_{2}(s) \mathrm{d} s+a_{2}\right)^{2}}{\left(1+\left\|u_{2}(t)\right\|^{2}\right)^{2}}\right| \\
& \leq C|y(x, t)|+\left|u_{2}(x, t)\right|\left[\left(1+\left\|u_{1}(t)\right\|^{2}\right)\left(\int_{0}^{t}\left|\gamma_{1}(s)-\gamma_{2}(s)\right| \mathrm{d} s+\left|a_{1}-a_{2}\right|\right)\right. \\
& \left.+\left(\int_{0}^{1}\left|u_{1}^{2}(x, t)-u_{2}^{2}(x, t)\right| \mathrm{d} x\right)\left(\int_{0}^{t} \gamma_{1}(s) \mathrm{d} s+a_{1}\right)\right] \\
& \times \frac{\left(1+\left\|u_{2}(t)\right\|^{2}\right)\left(\int_{0}^{t} \gamma_{1}(s) \mathrm{d} s+a_{1}\right)+\left(1+\left\|u_{1}(t)\right\|^{2}\right)\left(\int_{0}^{t} \gamma_{2}(s) \mathrm{d} s+a_{2}\right)}{\left(1+\left\|u_{1}(t)\right\|^{2}\right)^{2}\left(1+\left\|u_{2}(t)\right\|^{2}\right)^{2}} \\
& \leq C|y(x, t)|+C\left[\left(1+\left\|u_{1}(t)\right\|^{2}\right)\left(\int_{0}^{t}\left|\gamma_{1}(s)-\gamma_{2}(s)\right| \mathrm{d} s+\left|a_{1}-a_{2}\right|\right)\right. \\
& \left.+\left\|u_{1}(t)-u_{2}(t)\right\|\left\|u_{1}(t)+u_{2}(t)\right\|\right]\left|u_{2}(x, t)\right| \\
& \times \frac{\left(1+\left\|u_{2}(t)\right\|^{2}\right)+\left(1+\left\|u_{1}(t)\right\|^{2}\right)}{\left(1+\left\|u_{1}(t)\right\|^{2}\right)^{2}\left(1+\left\|u_{2}(t)\right\|^{2}\right)^{2}} \\
& \leq C|y(x, t)|+\frac{C\left(\|y(t)\|+\int_{0}^{t}\left|\gamma_{1}(s)-\gamma_{2}(s)\right| \mathrm{d} s+\left|a_{1}-a_{2}\right|\right)\left|u_{2}(x, t)\right|}{\left(1+\left\|u_{2}(t)\right\|^{2}\right)^{1 / 2}},
\end{aligned}
$$


we deduce that

$$
\begin{aligned}
\frac{\mathrm{d}}{\mathrm{d} t}\left(\left\|y_{t}(t)\right\|^{2}+\left\|y_{x x}(t)\right\|^{2}\right) & \leq 2 \int_{0}^{1}\left|f\left(t, u_{1}(x, t), \gamma_{1}, a_{1}\right)-f\left(t, u_{2}(x, t), \gamma_{2}, a_{2}\right) \| y_{t}(x, t)\right| \mathrm{d} x \\
& \leq C\left(\left\|y_{t}(t)\right\|^{2}+\|y(t)\|^{2}+\left\|\gamma_{1}-\gamma_{2}\right\|_{L^{1}(0, T)}^{2}+\left|a_{1}-a_{2}\right|^{2}\right) \\
& \leq C\left(\left\|y_{t}(t)\right\|^{2}+\left\|y_{x x}(t)\right\|^{2}+\left\|\gamma_{1}-\gamma_{2}\right\|_{L^{1}(0, T)}^{2}+\left|a_{1}-a_{2}\right|^{2}\right)
\end{aligned}
$$

It therefore follows from Gronwall's inequality (see, e.g. [14], p. 90) that for $0 \leq t \leq T$

$$
\left\|u_{1 x x}(t)-u_{2 x x}(t)\right\|+\left\|u_{1 t}(t)-u_{2 t}(t)\right\| \leq C\left(\left\|u_{1 x x}^{0}-u_{2 x x}^{0}\right\|+\left\|u_{1}^{1}-u_{2}^{1}\right\|+\left|\omega_{1}^{0}-\omega_{2}^{0}\right|+\left\|\gamma_{1}-\gamma_{2}\right\|_{L^{1}(0, T)}\right)
$$

Furthermore, since

$$
\begin{aligned}
\left|\omega_{1}(t)-\omega_{2}(t)\right| & =\left|\frac{\int_{0}^{t} \gamma_{1}(s) \mathrm{d} s+a_{1}}{1+\left\|u_{1}(t)\right\|^{2}}-\frac{\int_{0}^{t} \gamma_{2}(s) \mathrm{d} s+a_{2}}{1+\left\|u_{2}(t)\right\|^{2}}\right| \\
& \leq C\left(\left\|u_{1}(t)-u_{2}(t)\right\|+\left\|u_{1 x x}^{0}-u_{2 x x}^{0}\right\|+\left\|u_{1}^{1}-u_{2}^{1}\right\|+\left|\omega_{1}^{0}-\omega_{2}^{0}\right|+\left\|\gamma_{1}-\gamma_{2}\right\|_{L^{1}(0, T)}\right)
\end{aligned}
$$

equation (2.7) follows from (2.27).

(ii) By Theorem 1.6 of [12] (p. 189), to prove that problem (1.1-1.4) has a unique strong solution for the initial condition $\left(u^{0}, u^{1}, \omega^{0}\right) \in H_{0^{-}}^{4}(0,1) \times H_{0^{-}}^{2}(0,1) \times \mathbb{R}$ and $\gamma \in C[0, T]$, it suffices to prove that, for any fixed $\gamma \in C[0, T]$ and $a_{0}, f:[0, T] \times L^{2}(0,1) \rightarrow L^{2}(0,1)$ is Lipschitz continuous in both variables $t$ and $\varphi$. This is true since

$$
\begin{aligned}
\left\|f\left(t_{1}, \varphi_{1}, \gamma, a_{0}\right)-f\left(t_{2}, \varphi_{2}, \gamma, a_{0}\right)\right\|= & \left\|\frac{\left(\int_{0}^{t_{1}} \gamma(s) \mathrm{d} s+a_{0}\right)^{2} \varphi_{1}(x)}{\left(1+\left\|\varphi_{1}\right\|^{2}\right)^{2}}-\frac{\left(\int_{0}^{t_{2}} \gamma(s) \mathrm{d} s+a_{0}\right)^{2} \varphi_{2}(x)}{\left(1+\left\|\varphi_{2}\right\|^{2}\right)^{2}}\right\| \\
\leq & \left(\int_{0}^{t_{2}}|\gamma(s)| \mathrm{d} s+\left|a_{0}\right|\right)^{2} \frac{\left\|\varphi_{1}(x)\left(1+\left\|\varphi_{2}\right\|^{2}\right)^{2}-\varphi_{2}(x)\left(1+\left\|\varphi_{1}\right\|^{2}\right)^{2}\right\|}{\left(1+\left\|\varphi_{1}\right\|^{2}\right)^{2}\left(1+\left\|\varphi_{2}\right\|^{2}\right)^{2}} \\
& +\frac{\left\|\varphi_{1}(x)\right\|}{\left(1+|| \varphi_{1} \|^{2}\right)^{2}\left|\int_{t_{1}}^{t_{2}} \gamma(s) \mathrm{d}\right|\left(\int_{0}^{t_{1}}|\gamma(s)| \mathrm{d} s+\int_{0}^{t_{2}}|\gamma(s)| \mathrm{d}+2\left|a_{0}\right|\right)} \\
\leq & \left(\int_{0}^{t_{2}}|\gamma(s)| \mathrm{d} s+\left|a_{0}\right|\right)^{2} \frac{\left\|\varphi_{1}-\varphi_{2}\right\|}{\left(1+\left\|\varphi_{1}\right\|^{2}\right)^{2}} \\
& +\left(\int_{0}^{t_{2}}|\gamma(s)| \mathrm{d} s+\left|a_{0}\right|\right)^{2} \frac{\left\|\varphi_{2}\right\|\left|\left\|\varphi_{2}\right\|^{2}-\left\|\varphi_{1}\right\|^{2}\right|\left(2+\left\|\varphi_{2}\right\|^{2}+\left\|\varphi_{1}\right\|^{2}\right)}{\left(1+\left\|\varphi_{1}\right\|^{2}\right)^{2}\left(1+\left\|\varphi_{2}\right\|^{2}\right)^{2}} \\
& +\left|\int_{t_{1}}^{t_{2}} \gamma(s) \mathrm{d} s\right|\left(\int_{0}^{t_{1}}|\gamma(s)| \mathrm{d} s+\int_{0}^{t_{2}}|\gamma(s)| \mathrm{d} s+2\left|a_{0}\right|\right) \\
\leq & C\left(\left|t_{2}-t_{1}\right|+\left\|\varphi_{1}-\varphi_{2}\right\|\right),
\end{aligned}
$$

where $C=C\left(\|\gamma\|_{L^{1}(0, T)},\|\gamma\|_{C[0, T]},\left|a_{0}\right|\right)$ is a positive constant independent of $\varphi_{1}$ and $\varphi_{2}$. 
Remark 2.1. Part (ii) of Theorem 2.1 will be used in the next section. When we prove a result, we will first assume that the initial data are smooth and then the equations have a strong solution so that computations are valid. We then apply a density argument in the case that the initial data are in $\mathcal{H}$.

\section{Optimal CONTROL}

This section is devoted to optimal control problem (1.8). The difficulty in dealing with the problem is that we can not prove whether the cost function $J(\gamma)$ is convex. It appears quite possible that $J(\gamma)$ is not. Thus we have to show that $J(\gamma)$ is weakly lower semi-continuous and then use a minimizing sequence to show the existence of an optimal control.

Theorem 3.1. For the initial condition $\left(u^{0}, u^{1}, \omega^{0}\right) \in \mathcal{H}$, problem (1.8) has at least one solution $\gamma^{*}$. Moreover, $\gamma^{*}$ can be characterized by

$$
\begin{gathered}
\int_{0}^{T}\left(\gamma(s)-\gamma^{*}(s)\right)\left[\gamma^{*}(s)+\int_{s}^{T} \frac{\int_{0}^{1} p(x, t) u^{*}(x, t) \mathrm{d} x\left(\int_{0}^{t} \gamma^{*}(\tau) \mathrm{d} \tau+a_{0}\right)}{\left(1+\left\|u^{*}(t)\right\|^{2}\right)^{2}} \mathrm{~d} t\right] \mathrm{d} s \\
+\int_{0}^{T}\left(\gamma(s)-\gamma^{*}(s)\right) \int_{s}^{T} \frac{\int_{0}^{t} \gamma^{*}(\tau) \mathrm{d} \tau+a_{0}-\bar{\omega}\left(1+\left\|u^{*}(t)\right\|^{2}\right)}{\left(1+\left\|u^{*}(t)\right\|^{2}\right)^{2}} \mathrm{~d} t \mathrm{~d} s \geq 0
\end{gathered}
$$

for all $\gamma \in \Gamma$, where $u^{*}$ is a solution of (2.14-2.16) corresponding to $\gamma^{*}$, and $p$ is the solution of

$$
\begin{aligned}
& p_{t t}(x, t)+p_{x x x x}(x, t)=h\left(x, t, \gamma^{*}, u^{*}, a_{0}, p\right), \\
& p(0, t)=p_{x}(0, t)=p_{x x}(1, t)=p_{x x x}(1, t)=0, \\
& p(x, T)=0, p_{t}(x, T)=0,
\end{aligned}
$$

with

$$
\begin{aligned}
h= & h\left(x, t, \gamma^{*}, u^{*}, a_{0}, p\right) \\
= & 2 u^{*}(x, t)+\frac{p(x, t)\left(\int_{0}^{t} \gamma^{*}(s) \mathrm{d} s+a_{0}\right)^{2}}{\left(1+\left\|u^{*}(t)\right\|^{2}\right)^{2}} \\
& -\frac{4 u^{*}(x, t)\left(\int_{0}^{t} \gamma^{*}(s) \mathrm{d} s+a_{0}\right)}{\left(1+\left\|u^{*}(t)\right\|^{2}\right)^{2}}\left(\frac{\int_{0}^{t} \gamma^{*}(s) \mathrm{d} s+a_{0}}{1+\left\|u^{*}(t)\right\|^{2}}-\bar{\omega}\right) \\
& -\frac{4 u^{*}(x, t)\left(\int_{0}^{t} \gamma^{*}(s) \mathrm{d} s+a_{0}\right)^{2} \int_{0}^{1} p(x, t) u^{*}(x, t) \mathrm{d} x}{\left(1+\left\|u^{*}(t)\right\|^{2}\right)^{3}} .
\end{aligned}
$$

Furthermore, if $\Gamma=L^{2}(0, T)$, then $\gamma^{*}$ satisfies

$$
\gamma^{*}(s)+\int_{s}^{T} \frac{\int_{0}^{1} p(x, t) u^{*}(x, t) \mathrm{d} x\left(\int_{0}^{t} \gamma^{*}(\tau) \mathrm{d} \tau+a_{0}\right)}{\left(1+\left\|u^{*}(t)\right\|^{2}\right)^{2}} \mathrm{~d} t+\int_{s}^{T} \frac{\int_{0}^{t} \gamma^{*}(\tau) \mathrm{d} \tau+a_{0}-\bar{\omega}\left(1+\left\|u^{*}(t)\right\|^{2}\right)}{\left(1+\left\|u^{*}(t)\right\|^{2}\right)^{2}} \mathrm{~d} t=0 .
$$

Remark 3.1. Whether the solution of (1.8) is unique or not is an open problem. However, we guess that the solution is not unique since for $\omega^{0}=0$ (then $a_{0}=0$ ) and $\bar{\omega}=0$ we have $J(-\gamma)=J(\gamma)$. 
Remark 3.2. Equation (3.6) shows that the optimal control $\gamma^{*} \in C^{1}[0, T]$. This is a sort of regularity property of the optimal control.

To prove this theorem, we first prove that the cost function $J(\gamma)$ is Gâteaux-differentiable and calculate its differential.

Lemma 3.1. The cost function $J(\gamma)$ is Gâteaux-differentiable. Furthermore, the differential at $\gamma_{o}$ in the direction $\gamma$ is given by

$$
\begin{aligned}
\left\langle J^{\prime}\left(\gamma_{0}\right), \gamma\right\rangle= & 2 \int_{0}^{T} \int_{0}^{1} u_{0}(x, t) \eta(x, t ; \gamma) \mathrm{d} x \mathrm{~d} t+2 \int_{0}^{T} \gamma_{0}(t) \gamma(t) \mathrm{d} t \\
& +2 \int_{0}^{T} \frac{\int_{0}^{t} \gamma(s) \mathrm{d} s}{1+\left\|u_{0}(t)\right\|^{2}}\left(\frac{\int_{0}^{t} \gamma_{0}(s) \mathrm{d} s+a_{0}}{1+\left\|u_{0}(t)\right\|^{2}}-\bar{\omega}\right) \mathrm{d} t \\
& -4 \int_{0}^{T} \frac{\int_{0}^{1} u_{0}(x, t) \eta(x, t ; \gamma) \mathrm{d} x\left(\int_{0}^{t} \gamma_{0}(s) \mathrm{d} s+a_{0}\right)}{\left(1+\left\|u_{0}(t)\right\|^{2}\right)^{2}}\left(\frac{\int_{0}^{t} \gamma_{0}(s) \mathrm{d} s+a_{0}}{1+\left\|u_{0}(t)\right\|^{2}}-\bar{\omega}\right) \mathrm{d} t
\end{aligned}
$$

where $u_{0}=u_{0}(x, t)$ is the solution of (2.14-2.16) corresponding to $\gamma_{0}$, and $\eta=\eta(x, t ; \gamma)$ is the solution of

$$
\begin{array}{ll}
\eta_{t t}(x, t)+\eta_{x x x x}(x, t)=g\left(x, t, \gamma_{0}, \gamma, a_{0}, \eta\right) & \text { in }(0,1) \times(0, T), \\
\eta(0, t)=\eta_{x}(0, t)=\eta_{x x}(1, t)=\eta_{x x x}(1, t)=0 & \text { in }(0, T), \\
\eta(x, 0)=0, \eta_{t}(x, 0)=0 & \text { in }(0,1),
\end{array}
$$

with

$$
\begin{aligned}
g= & g\left(x, t, \gamma_{0}, \gamma, a_{0}, \eta\right) \\
= & \frac{\eta(x, t)\left(\int_{0}^{t} \gamma_{0}(s) \mathrm{d} s+a_{0}\right)^{2}}{\left(1+\left\|u_{0}(t)\right\|^{2}\right)^{2}}+\frac{2 u_{0}(x, t)\left(\int_{0}^{t} \gamma_{0}(s) \mathrm{d} s+a_{0}\right) \int_{0}^{t} \gamma(s) \mathrm{d} s}{\left(1+\left\|u_{0}(t)\right\|^{2}\right)^{2}} \\
& -\frac{4 u_{0}(x, t)\left(\int_{0}^{t} \gamma_{0}(s) \mathrm{d} s+a_{0}\right)^{2} \int_{0}^{1} \eta u_{0} \mathrm{~d} x}{\left(1+\left\|u_{0}(t)\right\|^{2}\right)^{3}} .
\end{aligned}
$$

Proof. We may as well assume that the initial data and $\gamma$ are smooth since the general case can be handled by a density argument. For the smooth data, Theorem 2.1 ensures that the equations have a strong solution and then computations performed below are valid.

For $\lambda>0$ and $\gamma, \gamma_{0} \in C[0, T]$, let $u_{\lambda}$ and $u_{0}$ denote the solutions of (2.14-2.16) corresponding to $\gamma_{0}+\lambda \gamma$ and $\gamma_{0}$, respectively, and set

$$
\begin{aligned}
& y_{\lambda}=\frac{u_{\lambda}-u_{0}}{\lambda}, \\
& z_{\lambda}=y_{\lambda}-\eta
\end{aligned}
$$


We are going to prove that $\left(z_{\lambda}, z_{\lambda t}\right) \rightarrow(0,0)$ in $C\left([0, T] ; H^{2}(0,1)\right) \times C\left([0, T] ; L^{2}(0,1)\right)$ as $\lambda \rightarrow 0^{+}$. It is clear that $z_{\lambda}$ satisfies

$$
\begin{array}{ll}
z_{t t}(x, t)+z_{x x x x}(x, t)=\frac{f\left(t, u_{\lambda}, \gamma_{0}+\lambda \gamma, a_{0}\right)-f\left(t, u_{0}, \gamma_{0}, a_{0}\right)}{\lambda}-g & \text { in }(0,1) \times(0, T), \\
z(0, t)=z_{x}(0, t)=z_{x x}(1, t)=z_{x x x}(1, t)=0 & \text { in }(0, T), \\
z(x, 0)=0, z_{t}(x, 0)=0 & \text { in }(0,1) .
\end{array}
$$

To estimate $z_{\lambda}$, we first estimate $\frac{f\left(t, u_{\lambda}, \gamma_{0}+\lambda \gamma, a_{0}\right)-f\left(t, u_{0}, \gamma_{0}, a_{0}\right)}{\lambda}-g$. Set

$$
I(\varphi)=1+\|\varphi\|^{2}
$$

Then we have

$$
\begin{aligned}
& f\left(t, u_{\lambda}, \gamma_{0}+\lambda \gamma, a_{0}\right)-f\left(t, u_{0}, \gamma_{0}, a_{0}\right) \\
& =\frac{\left(\int_{0}^{t}\left(\gamma_{0}(s)+\lambda \gamma(s)\right) \mathrm{d} s+a_{0}\right)^{2} u_{\lambda}}{I^{2}\left(u_{\lambda}(t)\right)}-\frac{\left(\int_{0}^{t} \gamma_{0}(s) \mathrm{d} s+a_{0}\right)^{2} u_{0}}{I^{2}\left(u_{0}(t)\right)} \\
& =\frac{\left(\int_{0}^{t}\left(\gamma_{0}(s)+\lambda \gamma(s)\right) \mathrm{d} s+a_{0}\right)^{2}\left(u_{\lambda}-u_{0}\right)}{I^{2}\left(u_{\lambda}(t)\right)} \\
& +\left(\frac{\left(\int_{0}^{t}\left(\gamma_{0}(s)+\lambda \gamma(s)\right) \mathrm{d} s+a_{0}\right)^{2}}{I^{2}\left(u_{\lambda}(t)\right)}-\frac{\left(\int_{0}^{t} \gamma_{0}(s) \mathrm{d} s+a_{0}\right)^{2}}{I^{2}\left(u_{0}(t)\right)}\right) u_{0} \\
& =\lambda y_{\lambda} \frac{\left(\int_{0}^{t}\left(\gamma_{0}(s)+\lambda \gamma(s)\right) \mathrm{d} s+a_{0}\right)^{2}}{I^{2}\left(u_{\lambda}(t)\right)} \\
& +u_{0}\left[I\left(u_{0}(t)\right)\left(\int_{0}^{t}\left(\gamma_{0}(s)+\lambda \gamma(s)\right) \mathrm{d} s+a_{0}\right)-I\left(u_{\lambda}(t)\right)\left(\int_{0}^{t} \gamma_{0}(s) \mathrm{d} s+a_{0}\right)\right] \\
& \times \frac{\left[I\left(u_{0}(t)\right)\left(\int_{0}^{t}\left(\gamma_{0}(s)+\lambda \gamma(s)\right) \mathrm{d} s+a_{0}\right)+I\left(u_{\lambda}(t)\right)\left(\int_{0}^{t} \gamma_{0}(s) \mathrm{d} s+a_{0}\right)\right]}{I^{2}\left(u_{\lambda}(t)\right) I^{2}\left(u_{0}(t)\right)} \\
& =\lambda y_{\lambda} \frac{\left(\int_{0}^{t}\left(\gamma_{0}(s)+\lambda \gamma(s)\right) \mathrm{d} s+a_{0}\right)^{2}}{I^{2}\left(u_{\lambda}(t)\right)} \\
& +\lambda u_{0} I\left(u_{0}(t)\right) \int_{0}^{t} \gamma(s) \mathrm{d} s \\
& \times \frac{\left[I\left(u_{0}(t)\right)\left(\int_{0}^{t}\left(\gamma_{0}(s)+\lambda \gamma(s)\right) \mathrm{d} s+a_{0}\right)+I\left(u_{\lambda}(t)\right)\left(\int_{0}^{t} \gamma_{0}(s) \mathrm{d} s+a_{0}\right)\right]}{I^{2}\left(u_{\lambda}(t)\right) I^{2}\left(u_{0}(t)\right)} \\
& -\lambda u_{0} \int_{0}^{1} y_{\lambda}\left(u_{0}+u_{\lambda}\right) \mathrm{d} x\left(\int_{0}^{t} \gamma_{0}(s) \mathrm{d} s+a_{0}\right) \\
& \times \frac{\left[I\left(u_{0}(t)\right)\left(\int_{0}^{t}\left(\gamma_{0}(s)+\lambda \gamma(s)\right) \mathrm{d} s+a_{0}\right)+I\left(u_{\lambda}(t)\right)\left(\int_{0}^{t} \gamma_{0}(s) \mathrm{d} s+a_{0}\right)\right]}{I^{2}\left(u_{\lambda}(t)\right) I^{2}\left(u_{0}(t)\right)} .
\end{aligned}
$$


Set

$$
\begin{aligned}
I_{1}= & y_{\lambda} \frac{\left(\int_{0}^{t}\left(\gamma_{0}(s)+\lambda \gamma(s)\right) \mathrm{d} s+a_{0}\right)^{2}}{I^{2}\left(u_{\lambda}(t)\right)}-\frac{\eta\left(\int_{0}^{t} \gamma_{0}(s) \mathrm{d} s+a_{0}\right)^{2}}{I^{2}\left(u_{0}(t)\right)}, \\
I_{2}= & u_{0} I\left(u_{0}(t)\right) \int_{0}^{t} \gamma(s) \mathrm{d} s \\
& \times \frac{\left[I\left(u_{0}(t)\right)\left(\int_{0}^{t}\left(\gamma_{0}(s)+\lambda \gamma(s)\right) \mathrm{d} s+a_{0}\right)+I\left(u_{\lambda}(t)\right)\left(\int_{0}^{t} \gamma_{0}(s) \mathrm{d} s+a_{0}\right)\right]}{I^{2}\left(u_{\lambda}(t)\right) I^{2}\left(u_{0}(t)\right)} \\
& -\frac{2 u_{0}\left(\int_{0}^{t} \gamma_{0}(s) \mathrm{d} s+a_{0}\right) \int_{0}^{t} \gamma(s) \mathrm{d} s}{I^{2}\left(u_{0}(t)\right)}, \\
I_{3}= & -u_{0} \int_{0}^{1} y_{\lambda}\left(u_{0}+u_{\lambda}\right) \mathrm{d} x\left(\int_{0}^{t} \gamma_{0}(s) \mathrm{d} s+a_{0}\right) \\
& \times \frac{\left[I\left(u_{0}(t)\right)\left(\int_{0}^{t}\left(\gamma_{0}(s)+\lambda \gamma(s)\right) \mathrm{d} s+a_{0}\right)+I\left(u_{\lambda}(t)\right)\left(\int_{0}^{t} \gamma_{0}(s) \mathrm{d} s+a_{0}\right)\right]}{I^{2}\left(u_{\lambda}(t)\right) I^{2}\left(u_{0}(t)\right)} \\
& +\frac{4 u_{0}\left(\int_{0}^{t} \gamma_{0}(s) \mathrm{d} s+a_{0}\right)^{2} \int_{0}^{1} \eta u_{0} \mathrm{~d} x}{I^{3}\left(u_{0}(t)\right)} .
\end{aligned}
$$

It then follows from (3.18) that

$$
\frac{f\left(t, u_{\lambda}, \gamma_{0}+\lambda \gamma, a_{0}\right)-f\left(t, u_{0}, \gamma_{0}, a_{0}\right)}{\lambda}-g=I_{1}+I_{2}+I_{3} .
$$

We now want to estimate $I_{1}, I_{2}$ and $I_{3}$. Firstly, by (2.7), we obtain

$$
\left\|u_{\lambda}(t)-u_{0}(t)\right\| \leq \lambda C\left\|\gamma-\gamma_{0}\right\|_{L^{1}(0, T)}
$$

and

$$
\left\|y_{\lambda}(t)\right\| \leq C\left\|\gamma-\gamma_{0}\right\|_{L^{1}(0, T)}
$$

For $I_{1}$, we have

$$
I_{1}=y_{\lambda}\left[\frac{\left(\int_{0}^{t}\left(\gamma_{0}(s)+\lambda \gamma(s)\right) \mathrm{d} s+a_{0}\right)^{2}}{I^{2}\left(u_{\lambda}(t)\right)}-\frac{\left(\int_{0}^{t} \gamma_{0}(s) \mathrm{d} s+a_{0}\right)^{2}}{I^{2}\left(u_{0}(t)\right)}\right]+\frac{z_{\lambda}\left(\int_{0}^{t} \gamma_{0}(s) \mathrm{d} s+a_{0}\right)^{2}}{I^{2}\left(u_{0}(t)\right)}
$$

and then

$$
\left\|I_{1}(t)\right\| \leq C_{1}(\lambda)\left\|y_{\lambda}(t)\right\|+C_{2}\left\|z_{\lambda}(t)\right\|
$$


where

$$
\begin{aligned}
C_{1}(\lambda) & =C_{1}\left(\lambda, u_{0}, u_{\lambda}, \gamma, \gamma_{0}, a_{0}, T\right) \\
& =\max _{0 \leq t \leq T}\left|\frac{\left(\int_{0}^{t}\left(\gamma_{0}(s)+\lambda \gamma(s)\right) \mathrm{d} s+a_{0}\right)^{2}}{I^{2}\left(u_{\lambda}(t)\right)}-\frac{\left(\int_{0}^{t} \gamma_{0}(s) \mathrm{d} s+a_{0}\right)^{2}}{I^{2}\left(u_{0}(t)\right)}\right|, \\
C_{2} & =C_{2}\left(u_{0}, \gamma_{0}, a_{0}, T\right) \\
& =\max _{0 \leq t \leq T} \frac{\left(\int_{0}^{t}\left|\gamma_{0}(s)\right| \mathrm{d} s+\left|a_{0}\right|\right)^{2}}{I^{2}\left(u_{0}(t)\right)} .
\end{aligned}
$$

By (3.21), we deduce

$$
\lim _{\lambda \rightarrow 0} C_{1}(\lambda)=0 .
$$

For $I_{2}$, it follows from (3.21) that

$$
\lim _{\lambda \rightarrow 0} \max _{0 \leq t \leq T}\left\|I_{2}(t, \lambda)\right\|=0 .
$$

For $I_{3}$, we have

$$
\begin{aligned}
\left\|I_{3}(t, \lambda)\right\| \leq & \left\|u_{0}(t)\right\|\left\|z_{\lambda}(t)\right\|\left\|u_{0}(t)+u_{\lambda}(t)\right\|\left(\int_{0}^{t}\left|\gamma_{0}(s)\right| \mathrm{d} s+\left|a_{0}\right|\right) \\
& \times \frac{\left[I\left(u_{0}(t)\right)\left(\int_{0}^{t}\left|\gamma_{0}(s)+\lambda \gamma(s)\right| \mathrm{d} s+\left|a_{0}\right|\right)+I\left(u_{\lambda}(t)\right)\left(\int_{0}^{t}\left|\gamma_{0}(s)\right| \mathrm{d} s+\left|a_{0}\right|\right)\right]}{I^{2}\left(u_{\lambda}(t)\right) I^{2}\left(u_{0}(t)\right)} \\
+ & \| u_{0} \int_{0}^{1} \eta\left(u_{0}+u_{\lambda}\right) \mathrm{d} x\left(\int_{0}^{t} \gamma_{0}(s) \mathrm{d} s+a_{0}\right) \\
& \times \frac{\left[I\left(u_{0}(t)\right)\left(\int_{0}^{t}\left(\gamma_{0}(s)+\lambda \gamma(s)\right) \mathrm{d} s+a_{0}\right)+I\left(u_{\lambda}(t)\right)\left(\int_{0}^{t} \gamma_{0}(s) \mathrm{d} s+a_{0}\right)\right]}{I^{2}\left(u_{\lambda}(t)\right) I^{2}\left(u_{0}(t)\right)} \\
& -\frac{4 u_{0}\left(\int_{0}^{t} \gamma_{0}(s) \mathrm{d} s+a_{0}\right)^{2} \int_{0}^{1} \eta u_{0} \mathrm{~d} x}{I^{3}\left(u_{0}(t)\right)} \| \\
\leq & C_{3}\left\|z_{\lambda}(t)\right\|+C_{4}(\lambda),
\end{aligned}
$$

where

$$
\begin{aligned}
C_{3}= & C_{3}\left(\lambda, u_{0}, u_{\lambda}, \gamma, \gamma_{0}, a_{0}, T\right) \\
= & \max _{0 \leq t \leq T}\left\|u_{0}(t)\right\|\left\|u_{0}(t)+u_{\lambda}(t)\right\|\left(\int_{0}^{t}\left|\gamma_{0}(s)\right| \mathrm{d} s+\left|a_{0}\right|\right) \\
& \times \frac{\left[I\left(u_{0}(t)\right)\left(\int_{0}^{t}\left|\gamma_{0}(s)+\lambda \gamma(s)\right| \mathrm{d} s+\left|a_{0}\right|\right)+I\left(u_{\lambda}(t)\right)\left(\int_{0}^{t}\left|\gamma_{0}(s)\right| \mathrm{d} s+\left|a_{0}\right|\right)\right]}{I^{2}\left(u_{\lambda}(t)\right) I^{2}\left(u_{0}(t)\right)},
\end{aligned}
$$




$$
\begin{aligned}
C_{4}(\lambda)= & C_{4}\left(\lambda, \eta, u_{0}, u_{\lambda}, \gamma, \gamma_{0}, a_{0}, T\right) \\
= & \max _{0 \leq t \leq T} \| u_{0}(t) \int_{0}^{1} \eta\left(u_{0}+u_{\lambda}\right) \mathrm{d} x\left(\int_{0}^{t} \gamma_{0}(s) \mathrm{d} s+a_{0}\right) \\
& \times \frac{\left[I\left(u_{0}(t)\right)\left(\int_{0}^{t}\left(\gamma_{0}(s)+\lambda \gamma(s)\right) \mathrm{d} s+a_{0}\right)+I\left(u_{\lambda}(t)\right)\left(\int_{0}^{t} \gamma_{0}(s) \mathrm{d} s+a_{0}\right)\right]}{I^{2}\left(u_{\lambda}(t)\right) I^{2}\left(u_{0}(t)\right)} \\
& -\frac{4 u_{0}(t)\left(\int_{0}^{t} \gamma_{0}(s) \mathrm{d} s+a_{0}\right)^{2} \int_{0}^{1} \eta u_{0} \mathrm{~d} x}{I^{3}\left(u_{0}(t)\right)} \| .
\end{aligned}
$$

By (3.21), we deduce

$$
\lim _{\lambda \rightarrow 0} C_{4}(\lambda)=0
$$

Multiplying (3.14) by $z_{\lambda t}$ and integrating over $(0,1)$ by parts, it follows from $(3.22,3.24,3.27-3.29)$ and $(3.32)$ that

$$
\begin{aligned}
\frac{\mathrm{d}}{\mathrm{d} t}\left(\left\|z_{\lambda t}(t)\right\|^{2}+\left\|z_{\lambda x x}(t)\right\|^{2}\right) & =2 \int_{0}^{1}\left(I_{1}+I_{2}+I_{3}\right) z_{\lambda t} \mathrm{~d} s \\
& \leq C_{5}(\lambda)+C_{6}\left(\left\|z_{\lambda t}(t)\right\|^{2}+\left\|z_{\lambda x x}(t)\right\|^{2}\right)
\end{aligned}
$$

where $C_{5}(\lambda) \rightarrow 0$ as $\lambda \rightarrow 0^{+}$. It therefore follows from Gronwall's inequality that

$$
\left\|z_{\lambda t}(t)\right\|^{2}+\left\|z_{\lambda x x}(t)\right\|^{2} \leq C_{5}(\lambda) T e^{C_{6} t}, \quad \forall t \in[0, T] .
$$

Hence we have

$$
\lim _{\lambda \rightarrow 0^{+}}\left(\left\|z_{\lambda t}(t)\right\|^{2}+\left\|z_{\lambda x x}(t)\right\|^{2}\right)=0
$$

unformly for $t \in[0, T]$. Since

$$
\begin{aligned}
\frac{J\left(\gamma_{0}+\lambda \gamma\right)-J\left(\gamma_{0}\right)}{\lambda}= & \int_{0}^{T} \int_{0}^{1} \frac{u_{\lambda}^{2}(x, t)-u_{0}^{2}(x, t)+\left[\omega_{\lambda}(t)-\bar{\omega}\right]^{2}-\left[\omega_{0}(t)-\bar{\omega}\right]^{2}}{\lambda} \mathrm{d} x \mathrm{~d} t \\
& +\int_{0}^{T} \frac{\left(\gamma_{0}+\lambda \gamma\right)^{2}-\gamma_{0}^{2}}{\lambda} \mathrm{d} t \\
= & \int_{0}^{T} \int_{0}^{1}\left[2 u_{0}(x, t) y_{\lambda}(x, t)+\lambda y_{\lambda}^{2}(x, t)\right] \mathrm{d} x \mathrm{~d} t \\
& +\int_{0}^{T} \frac{1}{\lambda}\left(\frac{\int_{0}^{t}\left(\gamma_{0}(s)+\lambda \gamma(s)\right) \mathrm{d} s+a_{0}}{1+\left\|u_{\lambda}(t)\right\|^{2}}-\frac{\int_{0}^{t} \gamma_{0}(s) \mathrm{d} s+a_{0}}{1+\left\|u_{0}(t)\right\|^{2}}\right) \\
& \times\left(\frac{\int_{0}^{t}\left(\gamma_{0}(s)+\lambda \gamma(s)\right) \mathrm{d} s+a_{0}}{1+\left\|u_{\lambda}(t)\right\|^{2}}+\frac{\int_{0}^{t} \gamma_{0}(s) \mathrm{d} s+a_{0}}{1+\left\|u_{0}(t)\right\|^{2}}-2 \bar{\omega}\right) \mathrm{d} t \\
& +\int_{0}^{T}\left[2 \gamma_{0}(s) \gamma(s)+\lambda \gamma^{2}(s)\right] \mathrm{d} t
\end{aligned}
$$




$$
\begin{aligned}
= & \int_{0}^{T} \int_{0}^{1}\left[2 u_{0}(x, t) y_{\lambda}(x, t)+\lambda y_{\lambda}^{2}(x, t)\right] \mathrm{d} x \mathrm{~d} t \\
& +\int_{0}^{T} \frac{\left(1+\left\|u_{0}(t)\right\|^{2}\right) \int_{0}^{t} \gamma(s) \mathrm{d} s}{\left(1+\left\|u_{\lambda}(t)\right\|^{2}\right)\left(1+\left\|u_{0}(t)\right\|^{2}\right)} \\
& \times\left(\frac{\int_{0}^{t}\left(\gamma_{0}(s)+\lambda \gamma(s)\right) \mathrm{d} s+a_{0}}{1+\left\|u_{\lambda}(t)\right\|^{2}}+\frac{\int_{0}^{t} \gamma_{0}(s) \mathrm{d} s+a_{0}}{1+\left\|u_{0}(t)\right\|^{2}}-2 \bar{\omega}\right) \mathrm{d} t \\
& -\int_{0}^{T} \frac{\int_{0}^{1} y_{\lambda}\left(u_{\lambda}+u_{0}\right) \mathrm{d} x\left(\int_{0}^{t} \gamma_{0}(s) \mathrm{d} s+a_{0}\right)}{\left(1+\left\|u_{\lambda}(t)\right\|^{2}\right)\left(1+\left\|u_{0}(t)\right\|^{2}\right)} \\
& \times\left(\frac{\int_{0}^{t}\left(\gamma_{0}(s)+\lambda \gamma(s)\right) \mathrm{d} s+a_{0}}{1+\left\|u_{\lambda}(t)\right\|^{2}}+\frac{\int_{0}^{t} \gamma_{0}(s) \mathrm{d} s+a_{0}}{1+\left\|u_{0}(t)\right\|^{2}}-2 \bar{\omega}\right) \mathrm{d} t \\
& +\int_{0}^{T}\left[2 \gamma_{0}(s) \gamma(s)+\lambda \gamma^{2}(s)\right] \mathrm{d} t,
\end{aligned}
$$

it follows from (3.21) and (3.35) that

$$
\begin{aligned}
\left\langle J^{\prime}\left(\gamma_{0}\right), \gamma\right\rangle= & \lim _{\lambda \rightarrow 0^{+}} \frac{J\left(\gamma_{0}+\lambda \gamma\right)-J\left(\gamma_{0}\right)}{\lambda} \\
= & 2 \int_{0}^{T} \int_{0}^{1} u_{0}(x, t) \eta(x, t ; \gamma) \mathrm{d} x \mathrm{~d} t+2 \int_{0}^{T} \gamma_{0}(t) \gamma(t) \mathrm{d} t \\
& +2 \int_{0}^{T} \frac{\left(1+\left\|u_{0}(t)\right\|^{2}\right) \int_{0}^{t} \gamma(s) \mathrm{d} s-2 \int_{0}^{1} u_{0}(x, t) \eta(x, t ; \gamma) \mathrm{d} x\left(\int_{0}^{t} \gamma_{0}(s) \mathrm{d} s+a_{0}\right)}{\left(1+\left\|u_{0}(t)\right\|^{2}\right)^{2}} \\
& \times\left(\frac{\int_{0}^{t} \gamma_{0}(s) \mathrm{d} s+a_{0}}{1+\left\|u_{0}(t)\right\|^{2}}-\bar{\omega}\right) \mathrm{d} t .
\end{aligned}
$$

We then show that the cost function $J(\gamma)$ is weakly lower semi-continuous on $L^{2}(0, T)$ (for definition, see, e.g. [8], pp. 9-11).

Lemma 3.2. The cost function $J(\gamma)$ is weakly lower semi-continuous on $L^{2}(0, T)$.

Proof. Let $\left\{\gamma_{n}\right\}$ weakly converges to $\gamma_{0}$ in $L^{2}(0, T)$. Let $\left(u_{n}, \omega_{n}\right)$ and $\left(u^{*}, \omega^{*}\right)$ denote the solutions of $(1.1-1.4)$ corresponding to $\gamma_{n}$ and $\gamma_{0}$, respectively. By $(2.7)$, the sequence $\left\{u_{n}, u_{n t}, \omega_{n}\right\}$ is bounded in $C([0, T] ; \mathcal{H} \times \mathbb{R})$. Therefore, there exists a subsequence, still denoted by $\left\{u_{n}, u_{n t}, \omega_{n}\right\}$, converges to $\left(\tilde{u}, \tilde{u}_{t}, \tilde{\omega}\right)$ star-weakly in $C([0, T] ; \mathcal{H} \times \mathbb{R})$. Moreover, it follows from the compact embedding theorem (see, e.g. [13], Th. 3, p. 80) that there exists a subsequence, still denoted by $\left\{u_{n}\right\}$, strongly converges to $\tilde{u}$ in $C\left([0, T] ; L^{2}(0,1)\right)$. We now prove that $u^{*}=\tilde{u}$. We note that $u_{n}$ satisfies the following integral equation

$$
\left(u_{n}, u_{n t}\right)=S(t)\left(u^{0}, u^{1}\right)+\int_{0}^{t} S(t-s)\left(0, \frac{\int_{0}^{s} \gamma_{n}(\tau) \mathrm{d} \tau+a_{0}}{1+\left\|u_{n}(s)\right\|^{2}} u_{n}(s)\right) \mathrm{d} s .
$$


Taking

$$
\theta(t)= \begin{cases}1, & \text { if } 0 \leq t \leq s \\ 0, & \text { if } s<t \leq T\end{cases}
$$

we deduce that

$$
\lim _{n \rightarrow \infty} \int_{0}^{s} \gamma_{n}(\tau) \mathrm{d} \tau=\lim _{n \rightarrow \infty} \int_{0}^{T} \gamma_{n}(\tau) \theta(\tau) \mathrm{d} \tau=\int_{0}^{T} \gamma_{0}(\tau) \theta(\tau) \mathrm{d} \tau=\int_{0}^{s} \gamma_{0}(\tau) \mathrm{d} \tau
$$

since $\left\{\gamma_{n}\right\}$ weakly converges to $\gamma_{0}$ in $L^{2}(0, T)$. Letting $n \rightarrow \infty$ in (3.38), we obtain

$$
\left(\tilde{u}, \tilde{u}_{t}\right)=S(t)\left(u^{0}, u^{1}\right)+\int_{0}^{t} S(t-s)\left(0, \frac{\int_{0}^{s} \gamma_{0}(\tau) \mathrm{d} \tau+a_{0}}{1+\left\|\tilde{u}^{2}(s)\right\|^{2}} \tilde{u}(s)\right) \mathrm{d} s,
$$

which implies that $\tilde{u}=u^{*}$. Moreover, we have

$$
\tilde{\omega}(s)=\lim _{n \rightarrow \infty} \omega_{n}(s)=\lim _{n \rightarrow \infty} \frac{\int_{0}^{s} \gamma_{n}(\tau) \mathrm{d} \tau+a_{0}}{1+\left\|u_{n}^{2}(s)\right\|^{2}}=\frac{\int_{0}^{s} \gamma_{0}(\tau) \mathrm{d} \tau+a_{0}}{1+\left\|u^{*}(s)\right\|^{2}}=\omega^{*}(s)
$$

It therefore follows that

$$
\begin{aligned}
J\left(\gamma_{0}\right) & =\int_{0}^{T} \int_{0}^{1}\left[u^{2}\left(x, t, \gamma_{0}\right)+\left(\omega\left(t, \gamma_{0}\right)-\bar{\omega}\right)^{2}\right] \mathrm{d} x \mathrm{~d} t+\int_{0}^{T}\left|\gamma_{0}\right|^{2}(t) \mathrm{d} t \\
& \leq \lim _{n \rightarrow \infty} \int_{0}^{T} \int_{0}^{1}\left[u_{n}^{2}(x, t)+\left(\omega_{n}(t)-\bar{\omega}\right)^{2}\right] \mathrm{d} x \mathrm{~d} t+\liminf _{n \rightarrow \infty} \int_{0}^{T}\left|\gamma_{n}\right|^{2}(t) \mathrm{d} t \\
& \leq \liminf _{n \rightarrow \infty} J\left(\gamma_{n}\right) .
\end{aligned}
$$

Hence, $J(\gamma)$ is weakly lower semi-continuous on $L^{2}(0, T)$.

We are now ready to prove Theorem 3.1.

Proof of Theorem 3.1. Let $\gamma_{n} \in \Gamma$ be a minimizing sequence such that

$$
\lim _{n \rightarrow \infty} J\left(\gamma_{n}\right)=\inf _{\gamma \in \Gamma} J(\gamma)
$$

Then the sequence $\left\{\gamma_{n}\right\}$ is bounded in $L^{2}(0, T)$. Hence, there exists a subsequence, still denoted by $\left\{\gamma_{n}\right\}$, weakly converges to $\gamma^{*}$ in $L^{2}(0, T)$. By Lemma 3.2 , we deduce that

$$
J\left(\gamma^{*}\right) \leq \liminf _{n \rightarrow \infty} J\left(\gamma_{n}\right)=\inf _{\gamma \in \Gamma} J(\gamma)
$$

Hence, $\gamma^{*}$ is a solution of (1.8). 
Since, by Lemma 3.1, $J(\gamma)$ is Gâteaux-differentiable, it follows from Theorem 1.3 of [10] (p. 10) (or Props. 1.2 and 2.1 of $[8]$, pp. 35-36) that

$$
\begin{aligned}
& \left\langle J^{\prime}\left(\gamma^{*}\right), \gamma-\gamma^{*}\right\rangle \\
& =2 \int_{0}^{T} \gamma^{*}(t)\left(\gamma(t)-\gamma^{*}(t)\right) \mathrm{d} t+2 \int_{0}^{T} \int_{0}^{1} u^{*}(x, t) \eta\left(x, t ; \gamma-\gamma^{*}\right) \mathrm{d} x \mathrm{~d} t \\
& \quad+2 \int_{0}^{T} \frac{\left(1+\left\|u^{*}(t)\right\|^{2}\right) \int_{0}^{t}\left(\gamma(s)-\gamma^{*}(s)\right) \mathrm{d} s}{\left(1+\left\|u^{*}(t)\right\|^{2}\right)^{2}}\left(\frac{\int_{0}^{t} \gamma^{*}(s) \mathrm{d} s+a_{0}}{1+\left\|u^{*}(t)\right\|^{2}}-\bar{\omega}\right) \mathrm{d} t \\
& \quad-4 \int_{0}^{T} \frac{\int_{0}^{1} u^{*}(x, t) \eta\left(x, t ; \gamma-\gamma^{*}\right) \mathrm{d} x\left(\int_{0}^{t} \gamma^{*}(s) \mathrm{d} s+a_{0}\right)}{\left(1+\left\|u^{*}(t)\right\|^{2}\right)^{2}}\left(\frac{\int_{0}^{t} \gamma^{*}(s) \mathrm{d} s+a_{0}}{1+\left\|u^{*}(t)\right\|^{2}}-\bar{\omega}\right) \mathrm{d} t \\
& \geq 0
\end{aligned}
$$

for all $\gamma \in \Gamma$. Multiplying (3.2) by $\eta$ and integrating over $(0,1) \times(0, T)$ by parts, we obtain

$$
\begin{aligned}
& \int_{0}^{T} \int_{0}^{1}\left(2 u^{*}+\frac{p\left(\int_{0}^{t} \gamma^{*}(s) \mathrm{d} s+a_{0}\right)^{2}}{\left(1+\left\|u^{*}(t)\right\|^{2}\right)^{2}}-\frac{4 u^{*}\left(\int_{0}^{t} \gamma^{*}(s) \mathrm{d} s+a_{0}\right)}{\left(1+\left\|u^{*}(t)\right\|^{2}\right)^{2}}\left(\frac{\int_{0}^{t} \gamma^{*}(s) \mathrm{d} s+a_{0}}{1+\left\|u^{*}(t)\right\|^{2}}-\bar{\omega}\right)\right. \\
& \left.-\frac{4 u^{*}\left(\int_{0}^{t} \gamma^{*}(s) \mathrm{d} s+a_{0}\right)^{2} \int_{0}^{1} p u^{*} \mathrm{~d} x}{\left(1+\left\|u^{*}(t)\right\|^{2}\right)^{3}}\right) \eta \mathrm{d} x \mathrm{~d} t \\
& =\int_{0}^{T} \int_{0}^{1} p\left(\eta_{t t}+\eta_{x x x x}\right) \mathrm{d} x \mathrm{~d} t \\
& =\int_{0}^{T} \int_{0}^{1} \frac{p \eta\left(\int_{0}^{t} \gamma^{*}(s) \mathrm{d} s+a_{0}\right)^{2}}{\left(1+\left\|u^{*}(t)\right\|^{2}\right)^{2}} \mathrm{~d} x \mathrm{~d} t \\
& +\int_{0}^{T} \int_{0}^{1} \frac{2 p u^{*}\left(\int_{0}^{t} \gamma^{*}(s) \mathrm{d} s+a_{0}\right) \int_{0}^{t}\left(\gamma(s)-\gamma^{*}(s)\right) \mathrm{d} s}{\left(1+\left\|u^{*}(t)\right\|^{2}\right)^{2}} \mathrm{~d} x \mathrm{~d} t \\
& -\int_{0}^{T} \int_{0}^{1} \frac{4 p u^{*}\left(\int_{0}^{t} \gamma^{*}(s) \mathrm{d} s+a_{0}\right)^{2} \int_{0}^{1} \eta u^{*} \mathrm{~d} x}{\left(1+\left\|u^{*}(t)\right\|^{2}\right)^{3}} \mathrm{~d} x \mathrm{~d} t .
\end{aligned}
$$


Hence we have

$$
\begin{aligned}
& \int_{0}^{T} \int_{0}^{1} u^{*} \eta \mathrm{d} x \mathrm{~d} t-2 \int_{0}^{T} \frac{\int_{0}^{1} u^{*} \eta \mathrm{d} x\left(\int_{0}^{t} \gamma^{*}(s) \mathrm{d} s+a_{0}\right)}{\left(1+\left\|u^{*}(t)\right\|^{2}\right)^{2}}\left(\frac{\int_{0}^{t} \gamma^{*}(s) \mathrm{d} s+a_{0}}{1+\left\|u^{*}(t)\right\|^{2}}-\bar{\omega}\right) \mathrm{d} t \\
& =\int_{0}^{T} \int_{0}^{1} \frac{p u^{*}\left(\int_{0}^{t} \gamma^{*}(s) \mathrm{d} s+a_{0}\right) \int_{0}^{t}\left(\gamma(s)-\gamma^{*}(s)\right) \mathrm{d} s}{\left(1+\left\|u^{*}(t)\right\|^{2}\right)^{2}} \mathrm{~d} x \mathrm{~d} t \\
& =\int_{0}^{T}\left(\gamma(s)-\gamma^{*}(s)\right) \int_{s}^{T} \frac{\int_{0}^{1} p(t) u^{*}(t) \mathrm{d} x\left(\int_{0}^{t} \gamma^{*}(\tau) \mathrm{d} \tau+a_{0}\right)}{\left(1+\left\|u^{*}(t)\right\|^{2}\right)^{2}} \mathrm{~d} t \mathrm{~d} s
\end{aligned}
$$

It therefore follows from (3.46) that

$$
\begin{aligned}
& \int_{0}^{T}\left(\gamma(s)-\gamma^{*}(s)\right)\left[\gamma^{*}(s)+\int_{s}^{T} \frac{\int_{0}^{1} p(x, t) u^{*}(x, t) \mathrm{d} x\left(\int_{0}^{t} \gamma^{*}(\tau) \mathrm{d} \tau+a_{0}\right)}{\left(1+\left\|u^{*}(t)\right\|^{2}\right)^{2}} \mathrm{~d} t\right] \mathrm{d} s \\
& \quad+\int_{0}^{T}\left(\gamma(s)-\gamma^{*}(s)\right) \int_{s}^{T} \frac{\left(1+\left\|u^{*}(t)\right\|^{2}\right)\left(\frac{\int_{0}^{t} \gamma^{*}(\tau) \mathrm{d} \tau+a_{0}}{1+\left\|u^{*}(t)\right\|^{2}}-\bar{\omega}\right)}{\left(1+\left\|u^{*}(t)\right\|^{2}\right)^{2}} \mathrm{~d} t \mathrm{~d} s \\
& \geq 0
\end{aligned}
$$

for all $\gamma \in \Gamma$. Hence, if $\Gamma=L^{2}(0, T)$, we deduce that

$$
\begin{gathered}
\gamma^{*}(s)+\int_{s}^{T} \frac{\int_{0}^{1} p(x, t) u^{*}(x, t) \mathrm{d} x\left(\int_{0}^{t} \gamma^{*}(\tau) \mathrm{d} \tau+a_{0}\right)}{\left(1+\left\|u^{*}(t)\right\|^{2}\right)^{2}} \mathrm{~d} t \\
+\int_{s}^{T} \frac{\left(1+\left\|u^{*}(t)\right\|^{2}\right)\left(\frac{\int_{0}^{t} \gamma^{*}(\tau) \mathrm{d} \tau+a_{0}}{1+\left\|u^{*}(t)\right\|^{2}}-\bar{\omega}\right)}{\left(1+\left\|u^{*}(t)\right\|^{2}\right)^{2}} \mathrm{~d} t=0 .
\end{gathered}
$$

We now turn to obstacle problems $(1.12,1.14)$ and $(1.16)$.

Theorem 3.2. Suppose the initial condition $\left(u^{0}, u^{1}, \omega^{0}\right) \in \mathcal{H}$ and let $\gamma^{*}$ be the solution of (1.8) with $\Gamma=$ $L^{2}(0, T)$. Then $\gamma^{*}$ is also a solution of problems (1.12) and (1.16).

Proof. Since

$$
J\left(\gamma^{*}\right)=\inf _{\gamma \in L^{2}(0, T)} J(\gamma) \leq F\left(\gamma^{*}\right)=\inf _{\gamma \in \Gamma\left(\gamma^{*}\right)} J(\gamma) \leq J\left(\gamma^{*}\right)
$$

we have

$$
J\left(\gamma^{*}\right)=F\left(\gamma^{*}\right)
$$

It therefore follows that

$$
F\left(\gamma^{*}\right)=J\left(\gamma^{*}\right) \leq \inf _{\gamma \in \Gamma(\theta)} J(\gamma)=F(\theta) \quad \forall \theta \in L^{2}(0, T)
$$


and

$$
H\left(\gamma^{*}\right)=F\left(\gamma^{*}\right)+J\left(\gamma^{*}\right) \leq F(\theta)+J(\theta)=H(\theta) \quad \forall \theta \in L^{2}(0, T)
$$

Theorem 3.3. For the initial condition $\left(u^{0}, u^{1}, \omega^{0}\right) \in \mathcal{H}$, problem (1.14) has at least one solution.

Proof. Let $\theta_{n} \in L^{2}(0, T)$ be a minimizing sequence such that

$$
\lim _{n \rightarrow \infty} G\left(\theta_{n}\right)=\inf _{\theta \in L^{2}(0, T)} G(\theta)
$$

By Theorem 3.1, there exists a $\gamma_{n} \in L^{2}(0, T)$ such that

$$
J\left(\gamma_{n}\right)=F\left(\theta_{n}\right) .
$$

Since

$$
G\left(\theta_{n}\right)=F\left(\theta_{n}\right)+\int_{0}^{T} \theta_{n}^{2}(t) \mathrm{d} s=J\left(\gamma_{n}\right)+\int_{0}^{T} \theta_{n}^{2}(t) \mathrm{d} s,
$$

the sequences $\left\{\theta_{n}\right\}$ and $\left\{\gamma_{n}\right\}$ are bounded in $L^{2}(0, T)$. Therefore there exist subsequences $\left\{\theta_{n_{i}}\right\}$ and $\left\{\gamma_{n_{i}}\right\}$ such that $\theta_{n_{i}}$ and $\gamma_{n_{i}}$ converge to $\bar{\theta}$ and $\gamma^{*}$ weakly in $L^{2}(0, T)$, respectively. It therefore follows from Lemma 3.2 that

$$
J\left(\gamma^{*}\right)+\|\bar{\theta}\|_{L^{2}(0, T)}^{2} \leq \liminf _{i \rightarrow \infty} J\left(\gamma_{n_{i}}\right)+\liminf _{i \rightarrow \infty}\left\|\theta_{n_{i}}\right\|_{L^{2}(0, T)}^{2} \leq \liminf _{i \rightarrow \infty} G\left(\theta_{n_{i}}\right)=\inf _{\theta \in L^{2}(0, T)} G(\theta) .
$$

Since

$$
\gamma_{n_{i}} \geq \theta_{n_{i}} \quad \text { a.e. on }(0, T),
$$

we deduce that for every $\phi \in L^{2}(0, T)$ with $\phi \geq 0$ a.e. on $(0, T)$

$$
\int_{0}^{T}\left(\gamma^{*}-\bar{\theta}\right) \phi \mathrm{d} t=\lim _{i \rightarrow \infty} \int_{0}^{T}\left(\gamma_{n_{i}}-\theta_{n_{i}}\right) \phi \mathrm{d} t \geq 0
$$

This shows that $\gamma^{*} \geq \bar{\theta}$ a.e. on $(0, T)$ and then $\gamma^{*} \in \Gamma(\bar{\theta})$. It therefore follows from (3.57) that

$$
G(\bar{\theta})=F(\bar{\theta})+\|\bar{\theta}\|_{L^{2}(0, T)}^{2} \leq J\left(\gamma^{*}\right)+\|\bar{\theta}\|_{L^{2}(0, T)}^{2} \leq \inf _{\theta \in L^{2}(0, T)} G(\theta) .
$$

\section{NUMERICAL SIMULATIONS}

On the basis of iteration method, we propose a numerical approximation scheme to calculate the optimal control. For a given control $\gamma$, we first solve equations $(2.14-2.16)$, which, by the difference method, can be 
discretized as follows

$$
\begin{aligned}
u_{i, 0}= & u^{0}(i / m), \quad i=0, \cdots, m, \\
u_{i, 1}= & u^{0}(i / m)+\delta u^{1}(i / m), \quad i=0, \cdots, m, \\
u_{0, j}= & 0, \quad j=0, \cdots, n, \\
u_{1, j}= & 0, \quad j=0, \cdots, n, \\
u_{i, j+1}= & -\left(u_{i-2, j}-4 u_{i-1, j}+6 u_{i, j}-4 u_{i+1, j}+u_{i+2, j}\right) \delta^{2} / h^{4}+2 u_{i, j}-u_{i, j-1} \\
& +\delta^{2} u_{i, j}\left(\int_{0}^{j \delta} \gamma(s) \mathrm{d} s+a_{0}\right)^{2} /\left(1+\|u(j \delta)\|^{2}\right)^{2}, \\
& i=2, \cdots, m-2, \quad j=1, \cdots, n-1, \\
u_{m-1, j+1}= & 2 u_{m-2, j+1}-u_{m-3, j+1}, \quad j=1, \cdots, n-1, \\
u_{m, j+1}= & 3 u_{m-2, j+1}-2 u_{m-3, j+1}, \quad j=1, \cdots, n-1,
\end{aligned}
$$

where $m$ and $n$ are positive integers and

$$
\begin{aligned}
h & =1 / m, \\
\delta & =T / n, \\
u_{i, j} & =u(i h, j \delta), \quad i=0, \cdots, m, \quad j=0, \cdots, n .
\end{aligned}
$$

The integrals $\int_{0}^{j \delta} \gamma(s) \mathrm{d} s$ and $\|u(j \delta)\|^{2}$ can be numerically calculated. In analogy, we then discretize the adjoint equations (3.2-3.4) and solve them by using the solution of (2.14-2.16). Finally, we solve control equation (3.6). For this, we set

$$
y(t)=\int_{0}^{t} \gamma(\tau) \mathrm{d} \tau
$$

Then (3.6) can be equivalently tranformed to

$$
y^{\prime \prime}-\frac{\int_{0}^{1} p(x, t) u^{*}(x, t) \mathrm{d} x+1}{\left(1+\left\|u^{*}(t)\right\|^{2}\right)^{2}} y=\frac{a_{0} \int_{0}^{1} p(x, t) u^{*}(x, t) \mathrm{d} x+a_{0}-\bar{\omega}\left(1+\left\|u^{*}(t)\right\|^{2}\right)}{\left(1+\left\|u^{*}(t)\right\|^{2}\right)^{2}}
$$

with $y(0)=0$ and $y^{\prime}(T)=0$. This equation can be numerically solved by the difference method. With this new control, we repeat the above procedure. The following numeric examples show that this scheme works very well. We take the initial data as follows

$$
\begin{aligned}
u^{0} & =100 x\left[(x-1)^{4}-1\right], \\
u^{1} & =-100 x\left[(x-1)^{4}-1\right], \\
\omega^{0} & =100.0, \\
\bar{\omega} & =1000.0
\end{aligned}
$$

and start iterations with three quite different initial controls

$$
\gamma_{0}=10000(3-t), \quad 0, \quad-10000 \sin (10 \pi(3-t)),
$$

respectively. In this case, the critical angular velocity $\omega_{c}=\sqrt{\mu_{1}} \approx 2.47$ according to Theorem 3 of [15], where $\mu_{1}$ is the smallest eigenvalue of the self-adjoint operator $\mathrm{d}^{4} / \mathrm{d} x^{4}$ with the domain $H_{0^{-}}^{4}(0,1)$. Thus $\bar{\omega}=1000.0$ is quite away from $\omega_{c}$. 
TABLE 4.1. Iteration procedures start with three quite different initial controls $\gamma_{0}=10000(3-$ $t), 0,-10000 \sin (10 \pi(3-t))$, respectively. Costs $J\left(\gamma_{i}\right)$ (defined by (1.6)) starting from each initial control tend to 5199745.1524 approximately and the iteration procedures are quite stable, where $\gamma_{i}$ is the control obtained at the $i$ th iteration.

\begin{tabular}{|l|l|l|l|}
\hline \multicolumn{4}{|c|}{$u^{0}=100 x\left[(x-1)^{4}-1\right], u^{1}=-u^{0}, \omega^{0}=100.0, \quad \bar{\omega}=1000.0$} \\
\hline \multirow{2}{*}{ Costs } & \multicolumn{3}{|c|}{ Initial controls $\gamma_{0}$ of iterations } \\
\cline { 2 - 4 } & $\gamma_{0}=10000(3-t)$ & \multicolumn{1}{|c|}{$\gamma_{0}=0$} & $\gamma_{0}=-10000 \sin (10 \pi(3-t))$ \\
\hline$J\left(\gamma_{0}\right)$ & $9.057124937905327 \mathrm{E} 8$ & 5168797.134532931 & $1.551708593258652 \mathrm{E} 8$ \\
\hline$J\left(\gamma_{1}\right)$ & 5518012.550900402 & $5199774.96504609)$ & 5200304.111160588 \\
\hline$J\left(\gamma_{2}\right)$ & 5198119.7475106 & 5199744.2495877575 & 5199752.349029758 \\
\hline$J\left(\gamma_{3}\right)$ & 5199730.987997696 & 5199745.147392863 & 5199745.2070749495 \\
\hline$J\left(\gamma_{4}\right)$ & 5199745.03486864 & 5199745.152405544 & 5199745.152939232 \\
\hline$J\left(\gamma_{5}\right)$ & 5199745.151464502 & 5199745.152431915 & 5199745.152484191 \\
\hline$J\left(\gamma_{6}\right)$ & 5199745.152485731 & 5199745.152409619 & 5199745.152427603 \\
\hline$J\left(\gamma_{7}\right)$ & 5199745.152470749 & 5199745.152436701 & 5199745.1523675015 \\
\hline$J\left(\gamma_{8}\right)$ & 5199745.152435771 & 5199745.152431848 & 5199745.152398524 \\
\hline$J\left(\gamma_{9}\right)$ & 5199745.152435782 & 5199745.1523830015 & 5199745.15244074 \\
\hline$J\left(\gamma_{10}\right)$ & 5199745.152427421 & 5199745.152397803 & 5199745.15244536 \\
\hline$J\left(\gamma_{11}\right)$ & 5199745.152365553 & 5199745.152396315 & 5199745.152459322 \\
\hline$J\left(\gamma_{12}\right)$ & 5199745.15240079 & 5199745.152411937 & 5199745.152441221 \\
\hline$J\left(\gamma_{13}\right)$ & 5199745.152441801 & 5199745.152458425 & 5199745.152410811 \\
\hline$J\left(\gamma_{14}\right)$ & 5199745.15244327 & 5199745.152439428 & 5199745.152403285 \\
\hline$J\left(\gamma_{15}\right)$ & 5199745.15244552 & 5199745.152435335 & 5199745.152392513 \\
\hline$J\left(\gamma_{16}\right)$ & 5199745.152474231 & 5199745.152455318 & 5199745.152425697 \\
\hline$J\left(\gamma_{17}\right)$ & 5199745.152458648 & 5199745.152434166 & 5199745.15244514 \\
\hline$J\left(\gamma_{18}\right)$ & 5199745.152442513 & 5199745.152427262 & 5199745.152393454 \\
\hline$J\left(\gamma_{19}\right)$ & 5199745.152466581 & 5199745.152467447 & 5199745.152394731 \\
\hline & & & \\
\hline
\end{tabular}

It can be seen from Table 4.1 that the costs $J\left(\gamma_{i}\right)$ (defined by (1.6)) starting from each initial control tend to 5199745.1524 approximately and the iteration procedures are quite stable, where $\gamma_{i}$ is the control obtained at the $i$-th iteration. Moreover, Figures 4.1, 4.2 and 4.3 show that all optimal controls and optimal solutions obtained from the iterations starting at these initial controls are almost same. In these figures, gamma denotes the control $\gamma(t), E$ is defined by

$$
E(t)=\int_{0}^{1} u^{2}(x, t) \mathrm{d} x,
$$

and omega the solution $\omega(t)$. The Java program for the simulations is available at http://www.mscs.dal.ca/ weiliu/beam/beamSimCtrl.java

\section{Conclusions}

In this paper we have proved the existence of an optimal control for the model of a rotating body beam, which is attached perpendicularly to the center of a rigid cylinder and rotates with the cylinder. The control is applied on the cylinder via a torque to suppress the vibrations of the beam. We have also derived a necessary condition for the control. Furthermore, on the basis of iteration method, we propose numerical approximation 

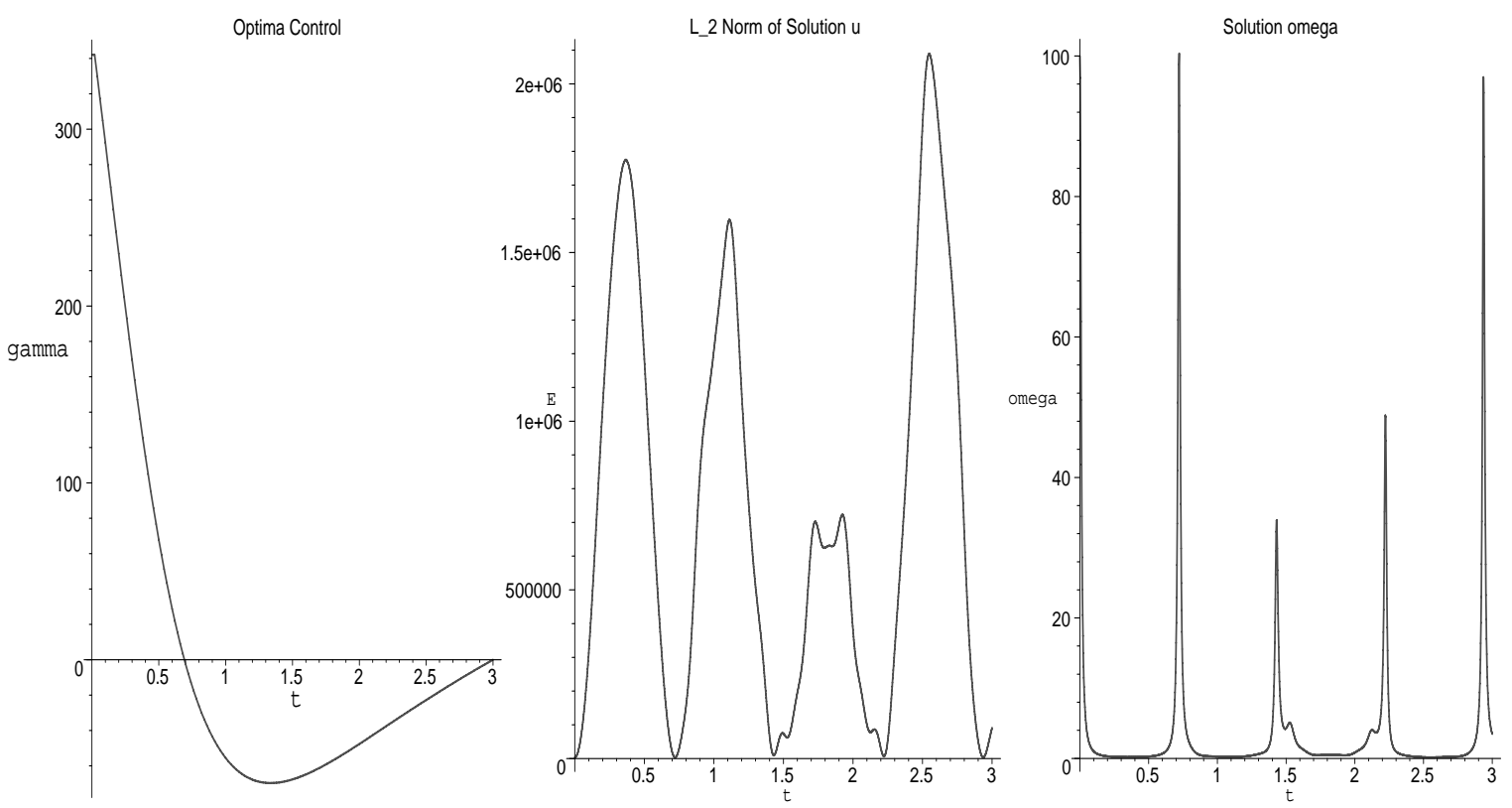

FigURE 4.1. Approximate optimal control and solution obtained via 19 iterations starting at $\gamma_{0}=10000(3-t)$.
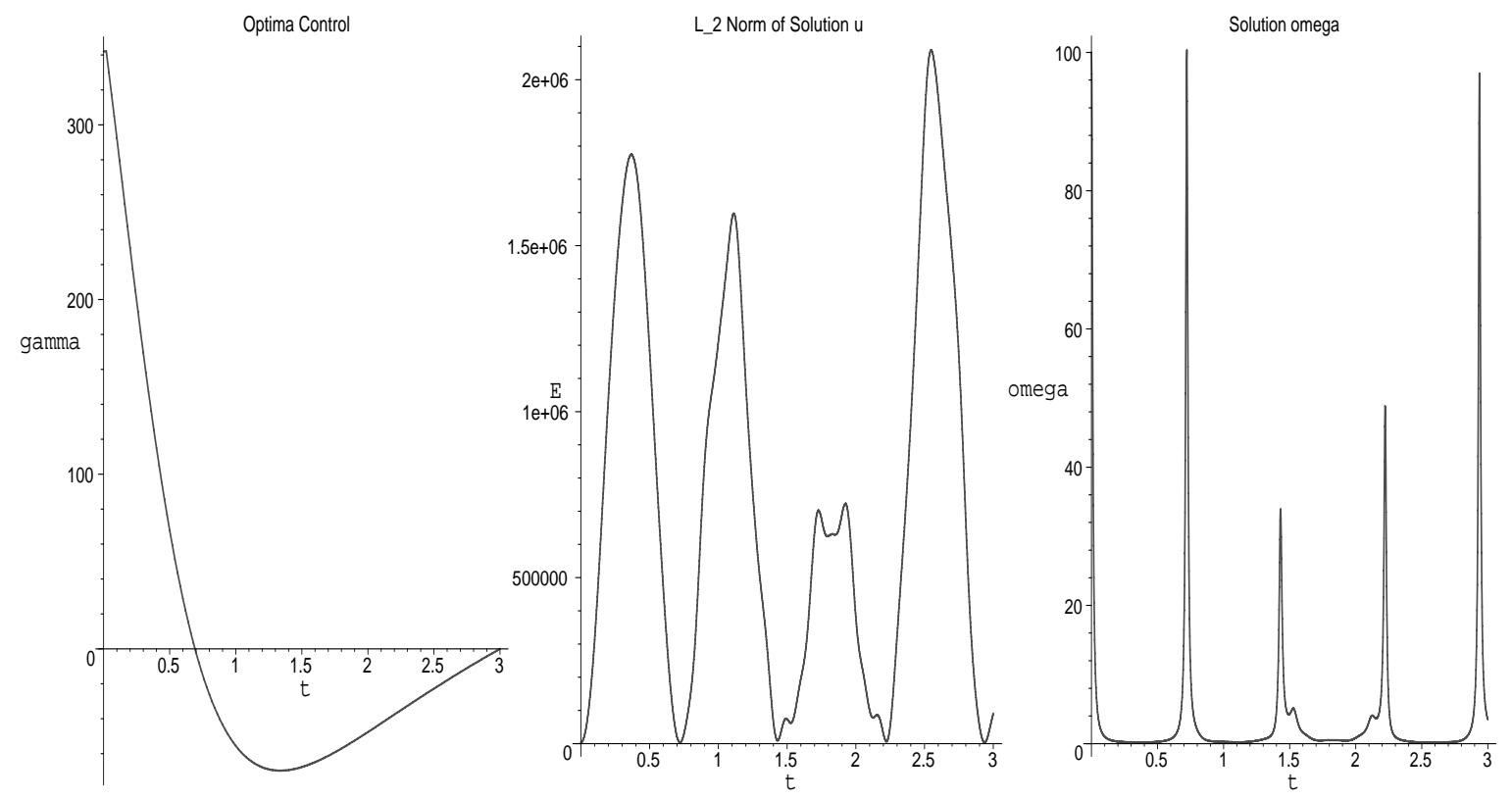

FiguRE 4.2. Approximate optimal control and solution obtained via 19 iterations starting at $\gamma_{0}=0$.

scheme to calculate the optimal control and give numeric examples to show that the scheme works well. Hence the control is implementable in real problems such as the control of a spacecraft.

In the study of stabilization for the beam via a feedback torque control, it is required that $\bar{\omega}$ of the equilibrium point $(0, \bar{\omega})$ be less than a critical angular velocity $\omega_{c}$. In our discussion of optimal control, this requirement is 

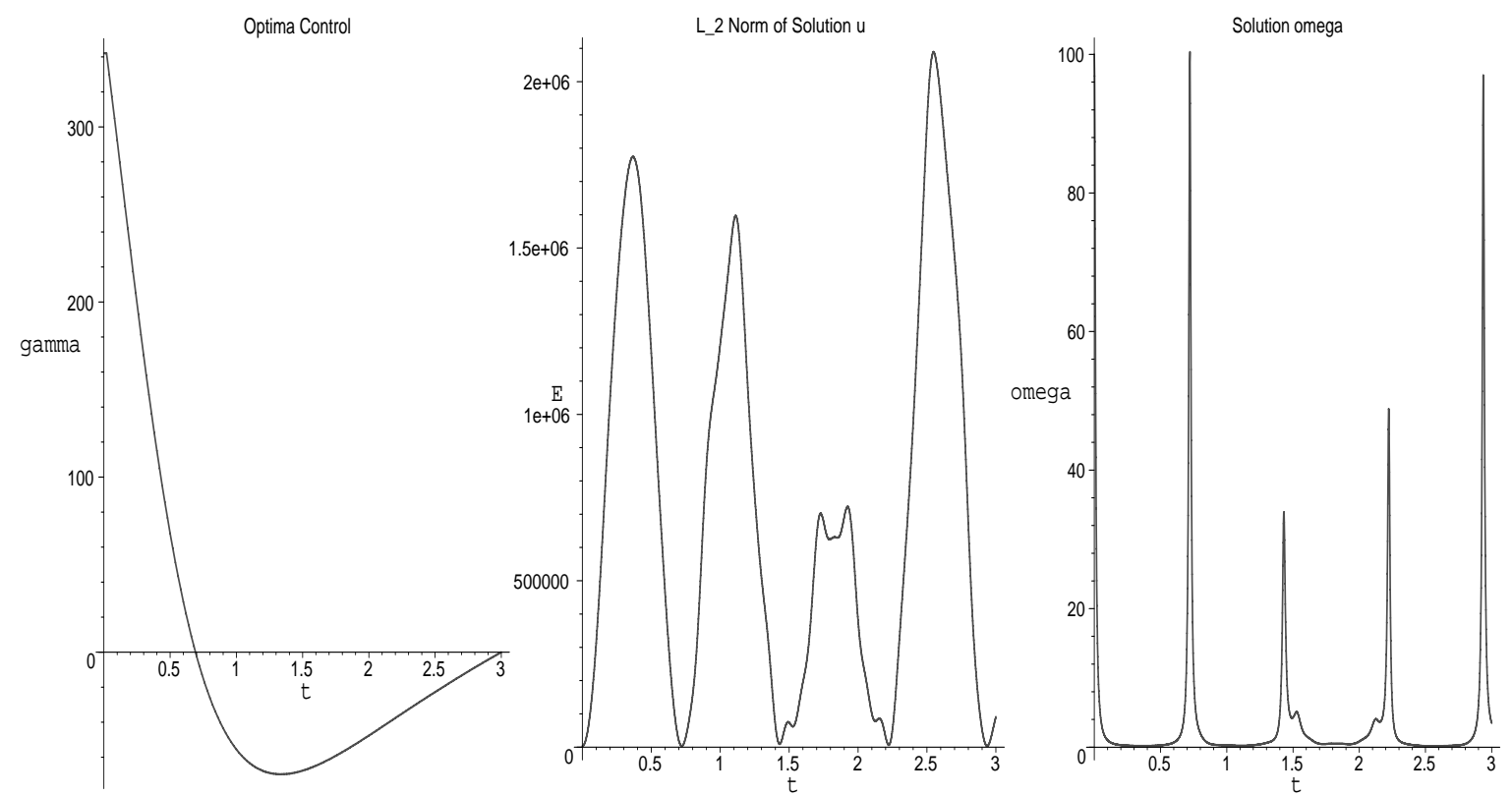

FigURE 4.3. Approximate optimal control and solution obtained via 19 iterations starting at $\gamma_{0}=-10000 \sin (10 \pi(3-t))$.

not needed. However, what we can achieve is less satisfactory, just driving the beam to approach its equilibrium 0 as closely as possible rather than exactly to its equilibrium as in the case of stabilization.

I thank the referees for their valuable criticisms and comments.

\section{REFERENCES}

[1] R. Adams, Sobolev Spaces. Academic Press, New York (1975).

[2] J. Baillieul and M. Levi, Rotational elastic dynamics. Physica D 27 (1987) 43-62.

[3] J. Baillieul and M. Levi, Constrained relative motions in rotational mechanics. Arch. Rational Mech. Anal. 115 (1991) 101-135.

[4] S.K. Biswas and N.U. Ahmed, Optimal control of large space structures governed by a coupled system of ordinary and partial differential equations. Math. Control Signals Systems 2 (1989) 1-18.

[5] B. Chentouf and J.F. Couchouron, Nonlinear feedback stabilization of a rotating body-beam without damping. ESAIM: COCV 4 (1999) 515-535.

[6] J.-M. Coron and B. d'Andréa-Novel, Stabilization of a rotating body beam without damping. IEEE Trans. Automat. Control 43 (1998) 608-618.

[7] C.J. Damaren and G.M.T. D'Eleuterio, Optimal control of large space structures using distributed gyricity. J. Guidance Control Dynam. 12 (1989) 723-731.

[8] I. Ekeland and R. Temam, Convex Analysis and Variational Problems. North-Holland Publishing Company, Amsterdam (1976).

[9] H. Laousy, C.Z. Xu and G. Sallet, Boundary feedback stabilization of a rotating body-beam system. IEEE Trans. Automat. Control 41 (1996) 241-245.

[10] J.L. Lions, Optimal Control of Systems Governed by Partial Differential Equations. Springer-Verlag, Berlin (1971).

[11] J.L. Lions and E. Magenes, Non-homogeneous Boundary value Problems and Applications, Vol. I. Springer-Verlag, Berlin, Heidelberg, New York (1972).

[12] A. Pazy, Semigroup of Linear Operators and Applications to Partial Differential Equations. Springer-Verlag, New York (1983).

[13] J. Simon, Compact sets in the space $L^{p}(0, T ; B)$. Ann. Mat. Pura Appl. (4) CXLVI (1987) 65-96.

[14] R. Temam, Infinite-dimensional Dynamical Systems in Mechanics and Physics, 2nd Ed. Springer-Verlag, New York (1997).

[15] C.Z. Xu and J. Baillieul, Stabilizability and stabilization of a rotating body-beam system with torque control. IEEE Trans. Automat. Control 38 (1993) 1754-1765. 